PAPER • OPEN ACCESS

\section{Response of nuclear track detector CR-39 to low energy muons}

To cite this article: H S P Thomas et al 2021 Plasma Phys. Control. Fusion 63124001

View the article online for updates and enhancements.
You may also like

- Fabrication of self-ruled micro grating on
$\frac{\text { CR-39 using ArF laser-induced rippling }}{\text { M Refahizadeh, P Parvin, K Silakhori et al. }}$
- Projection imaging with directional electron
$\frac{\text { and proton beams emitted from an }}{\text { ultrashort intense laser-driven thin foil }}$
$\frac{\text { target }}{\text { M Nishiuchi, I W Choi, H Daido et al. }}$
- Inter-comparison of radon detectors for
$\frac{\text { one to four week measurement periods }}{\text { G A Gunning, M Murray, S C Long et al. }}$




\title{
Response of nuclear track detector CR-39 to low energy muons
}

\author{
H S P Thomas ${ }^{1}$ (D, R M Deas ${ }^{1, *}$, L N Kirkham ${ }^{1}$, P M Dodd ${ }^{1}$, E Zemaityte $^{2,3}$, A D Hillier $^{4}$ \\ and D Neely ${ }^{3,5}$
}

${ }^{1}$ DSTL, Porton Down, Salisbury, Wiltshire SP4 0JQ, United Kingdom

${ }^{2}$ University of Strathclyde, Glasgow G12 8QQ, United Kingdom

${ }^{3}$ Central Laser Facility, STFC Rutherford Appleton Laboratory, Didcot OX11 0QX, United Kingdom

${ }^{4}$ ISIS Pulsed Neutron and Muon Source, STFC Rutherford Appleton Laboratory, Didcot OX11 0QX, United Kingdom

E-mail: rmdeas@dstl.gov.uk

Received 15 April 2021, revised 9 June 2021

Accepted for publication 9 September 2021

Published 20 October 2021

\begin{abstract}
The effectiveness of the PolyAllyl Diglycol Carbonate (PADC) etched solid state nuclear track detector (SSNTD), commonly known as CR-39, as a muon detector is assessed. CR-39 is successfully used to detect higher rest mass particles such as neutrons and protons, and is, for example, widely used in neutron dosimetry applications. CR-39 is generally accepted as being less suitable to detect lower rest mass particles such as muons, and especially electrons, due mostly to their reduced momenta and consequently, reduced stopping power. However, there has been some evidence that CR-39 may have application in the detection of cosmic ray muons. Monte Carlo simulations indicate that CR-39 can detect muons with energies up to $2.8 \mathrm{MeV}$. Experimental data to demonstrate the ability of CR-39 to detect muons was acquired using the MuSR spectrometer station at the ISIS Neutron and Muon Source. Pits deposited in CR-39 generated by positive muons from the beamline have been characterised and compared with pits deposited by protons and neutrons from other sources. The extent to which a CR-39 SSNTD can discriminate muons from particles with different momenta and rest masses is discussed.
\end{abstract}

Keywords: PADC, TASTRAK, CR-39, muons, muon detector

(Some figures may appear in colour only in the online journal)

\section{Introduction}

Conventional muon detectors are generally large scale installations, such as the compact muon solenoid system at the Large Hadron Collider, which consists of $2000 \mathrm{~m}^{3}$ of drift tubes [1]. A mini muon tracker system has been developed that is an

\footnotetext{
5 Died 27 August 2020.

Author to whom any correspondence should be addressed.
}

Original Content from this work may be used under the terms of the Creative Commons Attribution 4.0 licence. Any further distribution of this work must maintain attribution to the author(s) and the title of the work, journal citation and DOI. order of magnitude smaller, at $\sim 4 \mathrm{~m}^{3}$ [2]; however, this muon detector still uses drift tubes.

Drift tubes rely on charged particles and photons to create trails of gaseous ionisation between conductive plates. In environments where there are many different types of charged particles at high dose rates, it can be difficult to achieve unambiguous muon detection with drift tubes.

Within the last two decades, the continued improvement of ultra-intense laser systems has allowed high energy particle acceleration on a compact scale [3-5] to become a reality, opening up the potential for a much broader range of applications. One such application is the production of muons by laser-wakefield accelerated electrons [6-8]. However, most laser plasma acceleration experiments produce a variety of sub-atomic particles and ionising radiation in high doses, 
making the de-convolved measurement of a specific particle or photon in an energy range particularly challenging. This is especially the case for muons produced from laser wakefield experiments as their yields are predicted to be especially low until the next generation of ultra-intense lasers and wakefield accelerators are developed.

Laser plasma acceleration experiments need particle and radiation detector technology to be developed not only towards the particle or radiation to be detected, but also the ability to deconvolve the measurement from a the high radiological background produced as a result of the plasma acceleration. In order to measure and characterise the particles, radiation and beams a variety of diagnostics can be drawn upon, depending on the parameter being measured, e.g. spatial profile, spectral characteristics, timing properties, etc.

One type of diagnostic commonly used are solid state nuclear track detectors (SSNTDs), which can be used to detect individual particles. Typically the SSNTD is exposed, chemically etched and optically imaged to reveal the latent tracks of individual particles within the sensitivity range of the SSNTD. A commercially available SSNTD is a plastic more commonly known by its trademarked name, CR-39 (Columbia Resin \#39), chemical name PADC (PolyAllyl Diglycol Carbonate). It is used in a wide range of science and technology applications, from eyeglass lenses to inertial confinement fusion experiments [9]. Its response to protons [10], neutrons [11], alpha particles [12], and heavier ions [13] is well studied, and there are examples of its use to study the spectral properties of laser accelerated proton beams in particular [16]. CR-39 can be used to distinguish between different types of particles (within its sensitivity range), while also being insensitive to electromagnetic pulses [17] and relatively insensitive to X-rays [18], gamma-rays [19] and electrons [20]. This makes it particularly well suited for laser plasma experiments, where it exhibits a low sensitivity to the high background of electrons and x-rays usually present, allowing more specific measurements of protons and neutrons to be made.

Some of the advantages and versatility of CR-39 make it worthwhile to investigate its potential as a muon detector. This paper outlines the modelling, testing and characterisation performed to investigate the feasibility of CR-39 as a muon detector, categorise its use cases for muon detection and areas of future study

\section{Concept: background}

As a charged particle propagates through CR-39, it leaves a latent track of damaged molecular bonds as it deposits energy. To reveal the tracks, CR-39 is chemically etched in an alkali solution (typically sodium hydroxide). The surface of the plastic etches away at a slow bulk etch rate and regions of damage etch away at a faster rate, revealing the latent track as a 'pit' on the surface.

The dimensions of the pit is dependent on the incident particle (type and energy) and its angle of incidence, however the etch conditions (concentration, temperature and time) also effect the resultant track size. To account for the variation in etch conditions, the bulk etch length (i.e. the depth the undamaged surface has etched away by) is measured as the independent variable, and used to compare between data collected under different etch conditions (e.g. different temperatures, or different chemical etch solution concentrations). As an example, after a bulk etch length of $40 \mu \mathrm{m}$, a $5 \mathrm{MeV}$ proton typically produces a track diameter of $\sim 5 \mu \mathrm{m}$ [20]. The tracks are then observed with an optical microscope to determine their spatial properties.

As CR-39 is primarily used for neutron and charged particle dosimetry, there are many studies characterising the detector for given particles species and energy ranges, allowing the relationship between energy and particle track diameter to be well understood [9, 13, 19]. Characterisation of CR-39's response to relatively low $(<10 \mathrm{MeV})$ energy muons does not appear to have been investigated.

There has been evidence of muons produced from the cosmic ray background being detected by CR-39 [23, 24], but these results appear to have been at low flux and in the presence of other cosmic ray particles.

CR-39 is generally understood to be insensitive to cosmic ray muons, which tend to have an average energy at sea level in the $\mathrm{GeV}$ region. This study used the MuSR Spectrometer station at the ISIS Neutron and Muon Source, UK, which receives muons of $28 \mathrm{MeV} \mathrm{c}^{-1}$ momentum. It was hypothesised the CR-39 may be sensitive to muons of these lower energies.

\section{Experimental design}

In this experiment we used PADC SSNTD commonly known as CR-39 obtained from Track Analysis Systems Ltd under the tradename TASTRAK. The sensitivity of CR-39 to different particles can be described in terms of a material's stopping power: the rate at which a particle deposits energy as it travels through a material. The stopping power of particles travelling through CR-39 will depend on their type and energy. This is summarised in figure 1 which shows simulated data for the stopping power of CR-39 for protons and muons. For polymer damage to occur, sufficient energy must be deposited within a given range in CR-39, which is indicated by the material's stopping power. It is known from previous studies that $1-30 \mathrm{MeV}$ protons are able to deposit sufficient energy for damage to occur and tracks to develop with etching. The graph shows that the relatively higher energy protons deposit a similar amount of energy to relatively lower energy muons; therefore, it is expected that muons within this energy range will produce damage in CR-39. Consideration should also be made of the $2.2 \mu$ s average lifetime [15] of muons when depositing energy in CR-39. The decay products of positive muons are a positron, an electron neutrino and a muon antineutrino. However, in a similar fashion to electrons, these decay products are unlikely to deposit any significant energy in CR-39, including the energy from the photons produced as a result of positron annihilation events in CR-39.

Due to the stopping power of CR-39, provided it can detect muons, there is also the potential for discrimination between protons and muons (provided the energy range of the protons 


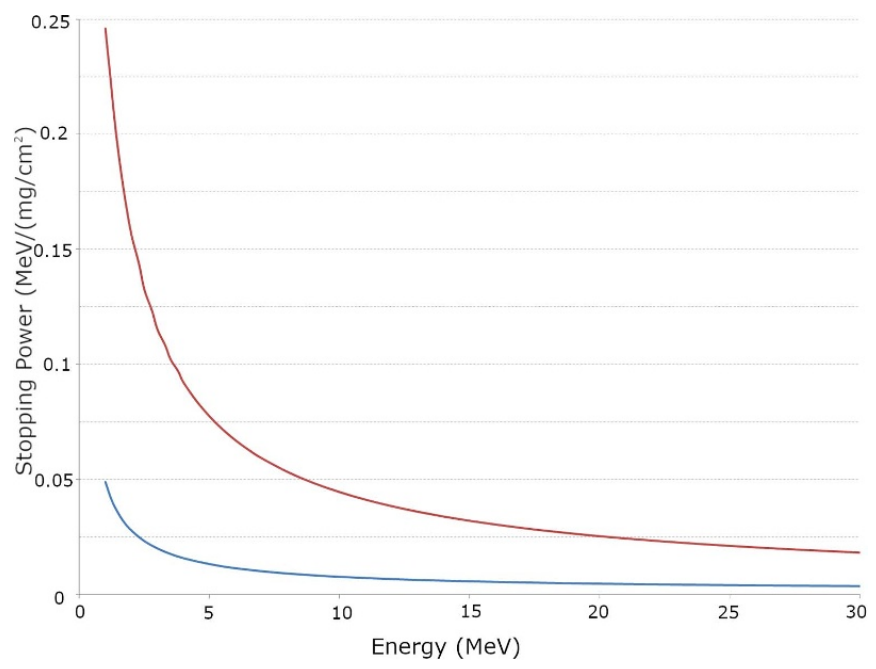

Figure 1. Monte Carlo Stopping and Range of Ions in Matter (SRIM) software simulated stopping power CR-39 for muons (shown on the graph in blue) and protons (shown on the graph in red).

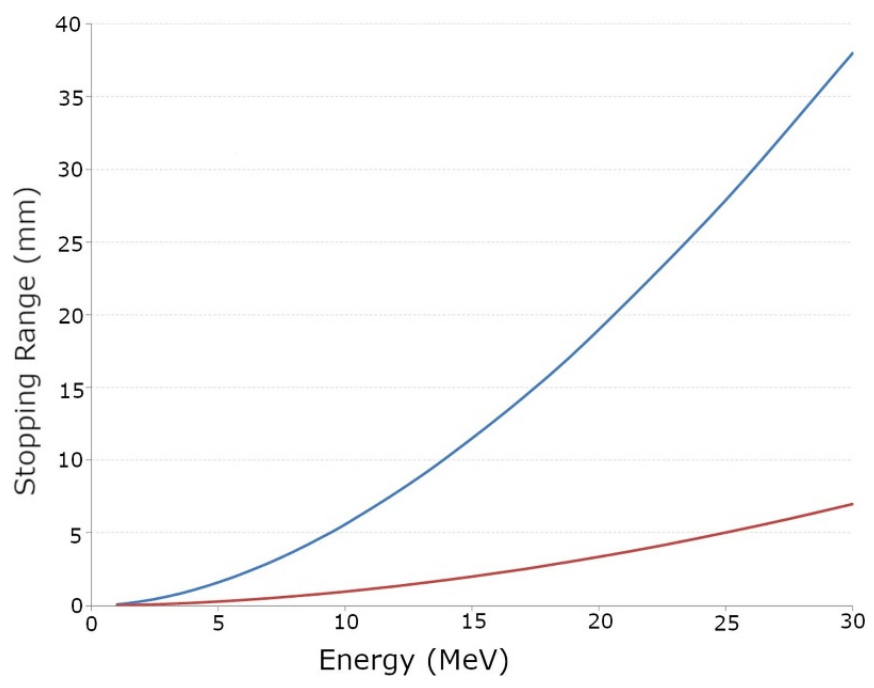

Figure 2. Stopping range of muons (shown on the graph in blue) and protons (shown on the graph in red) of different energies.

is known). Comparing the rate of decline of stopping power for the two particle types suggests that for similar energy particles, CR-39 has a greater stopping power for protons; i.e. a $5 \mathrm{MeV}$ proton will not travel as far through CR-39 as a $5 \mathrm{MeV}$ muon, as shown clearly in figure 2 . This difference in stopping power gives rise to a potential technique for distinguishing between the two particles over certain energy ranges in an environment where both are present, by identifying the depth in the CR-39 beyond which the protons should have lost their energy and will not be present.

Take for example, a $10 \mathrm{MeV}$ muon can travel up to $\sim 5 \mathrm{~mm}$ through CR-39, where a $10 \mathrm{MeV}$ proton should only travel up to $\sim 1 \mathrm{~mm}$. This stopping range was used to specifically design stacks to test the hypothesis that CR-39 would detect muons. For polymer damage to occur, sufficient energy must be deposited within a given range in the CR-39, which is indicated by the material's stopping power. From the literature, it is known that $1-30 \mathrm{MeV}$ protons are able to deposit sufficient energy for damage to occur and tracks to develop with etching. The stopping range graph (figure 2) shows that the relatively higher energy protons deposit a similar amount of energy to relatively lower energy muons; therefore, it is expected that muons within this energy range will produce damage in the CR-39.

Stacks were designed to detect muons across a range of energies, spanning the range they were predicted to deposit energy in. The stack of CR-39 was made up of multiple thin elements (200 $\mu \mathrm{m}$ thick), to give the most surfaces on which pits could be detected. This also gave the possibility of a higher chance of muons which were the correct energy for energy deposition, in the eventuality that it was only a very narrow range. To also provide a continuous varying thickness between these surfaces, two different wedges were also used on the front of the CR-39 stacks, as shown in figure 3. The two wedges were a plastic (PMMA) wedge, which tapered down from $1 \mathrm{~mm}$, and an aluminium wedge which tapered down from $600 \mu \mathrm{m}$. As the muon source used was quasimonoenergetic (with $2 \%$ bite) [14], this provided a continuous spectra of energies which ensured damage to the CR-39 would happen at a surface where it could be detected. A third stack was used as a background stack, placed within the area but not in path of the beam.

From Geant4 modelling (Monte Carlo simulations), given an initial energy of $3.88 \mathrm{MeV}$, a negligible amount of muons make it through $1000 \mu \mathrm{m}$ of CR-39, irrespective of where they are along the plastic or aluminium wedge. This informed the stack design, and the final design used was $2000 \mu \mathrm{m}$ total, a stack of 10 elements.

\section{Muon exposure}

These stacks of CR-39 were then exposed to the muons in the MuSR spectrometer at the ISIS Neutron and Muon Source.

The ISIS European Commission Muon Facility was accessed to provide a low-background source of quasimonoenergetic (with $2 \%$ bite) positive muons. The muon beam has a momentum of $28.63 \pm 0.66 \mathrm{MeV} \mathrm{c}^{-1}$, equivalent to an energy of $\sim 3.88 \mathrm{MeV}$, with a beam diameter of $\sim 18 \mathrm{~mm}$. The beam is pulsed at a rate of 40 pulses/sec with $\sim 20000$ muons/pulse produced [14].

Three stacks were used, as shown in figure 3 and described previously. Stack \#1 was placed in the target area, away from the beam, as a background measurement. Stacks \#2 and \#3 were exposed to the direct muon beam, one at a time. The beam was roughly centred on the centre of the front element and the exposure time was $\sim 10$ seconds, resulting in an exposure of $\sim 8 \times 10^{6}$ muons.

Post-exposure, the sheets of CR-39 were then etched in sodium hydroxide solution, of $6.25 \mathrm{M}$, heated to $85 \pm 5^{\circ} \mathrm{C}$ (the bulk etch length of this process is $4.25 \mu \mathrm{m} \mathrm{h}^{-1}$ ). Scanning was carried out with an automated scanning system and software produced by Track Analysis Systems Ltd (TASLIMAGE). The 

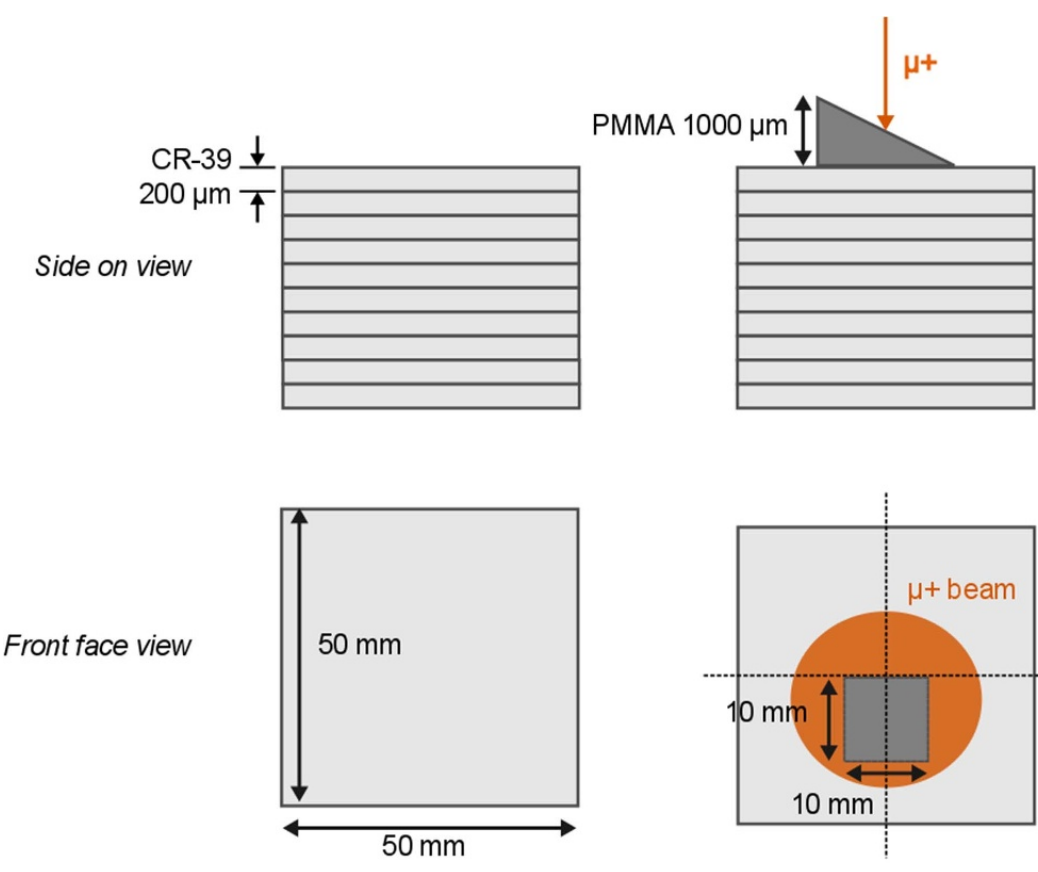

Stack\#1

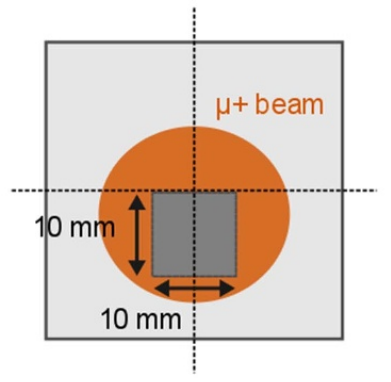

Stack \#2
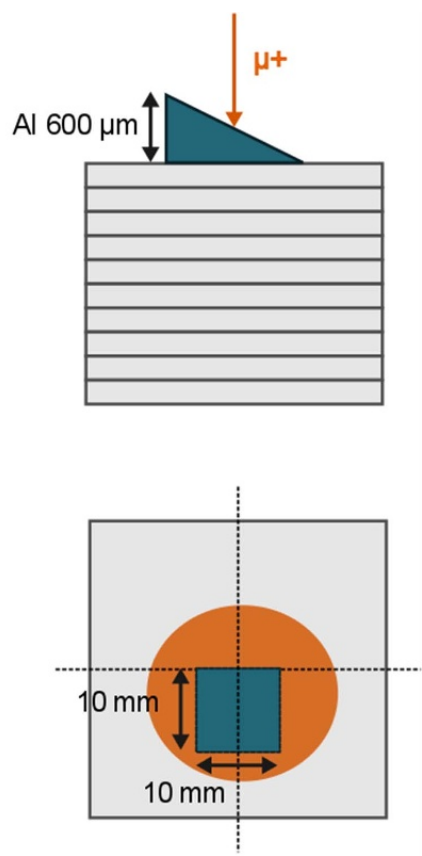

Stack \#3

Figure 3. The design of the three stacks used in ISIS. On the left hand side of the image, Stack \#1, consisted of 10 CR-39 elements, $50 \mathrm{~mm} \times 50 \mathrm{~mm}$ and each $200 \mu \mathrm{m}$ thick, stacked and placed in the area out of the direct beam as a background measurement. Stack \#2, in the centre, was the same as the background stack, but with an additional plastic polymethyl methacrylate (PMMA) wedge in front of it. Stack \#3, on the right hand side of the image, was again the same as the background stack but with an aluminium wedge in front of it.

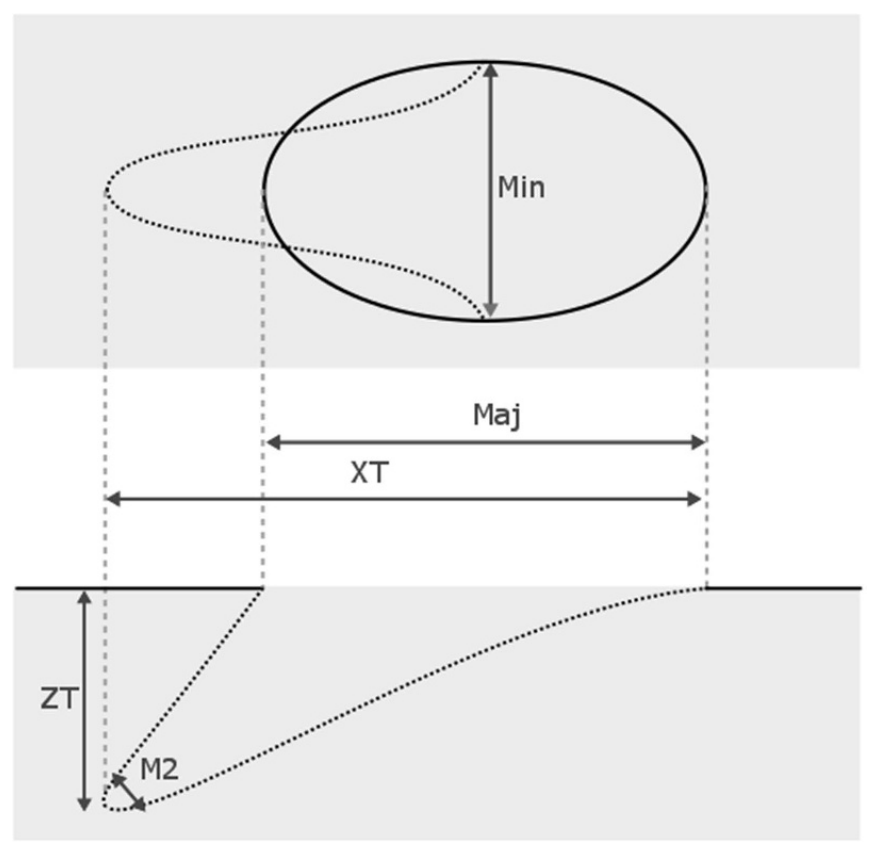

Figure 4. Measured parameters of accepted pits.

system consists of a motorised, backlit stage where an element is held, which is then translated within the image frame of a microscope. The CR-39 is clamped between two glass plates to mitigate any warping effects (a result of the decreased thickness due to etching), and to ensure that the microscope stays in focus as it images different areas of the surface.
The TASLIMAGE scanning and readout system [21] detects and records 'events' within a given frame, measuring the parameters shown in figure 4, such as: the minor and major diameter of each pit, the depth to which the track has been etched, and information which can give the angle of incidence of the track. These parameters are used to filter the data, and define whether the event is a true pit or a false detection.

There were various sources of image artefacts, such as scratches or residue from etching (despite washing in distilled water). Another artefact was due to the very thin CR-39 used, which meant pits created on the opposite side of the piece of CR-39 can be simultaneously imaged, albeit out of focus. These were not accepted as valid pits through filtering, as the edges were so out of focus the measured parameters would indicate a non-elliptical pit.

Figure 4 shows the pit parameters which were measured and estimated by the TASL software and so could be used to analyse the pits. These parameters were used to reject any pits with too steep an entry angle into the CR-39. As the CR-39 had been placed perpendicular to a collimated source of muons, this would reject any particles not associated with the source.

Figures 5 and 6 are of pieces of CR-39 taken from the same stack (Stack \#2, with the plastic wedge). Figure 5 shows the majority of muons are detected within the area beneath the plastic wedge. The wedge moderates the muons down to an energy at which they will deposit energy in the CR-39 and cause detectable tracks. Muons that do not pass through the wedge maintain their higher energies and pass through the CR-39 undetected. This piece is the backside of the 3rd element in the stack, so the muons have passed through 


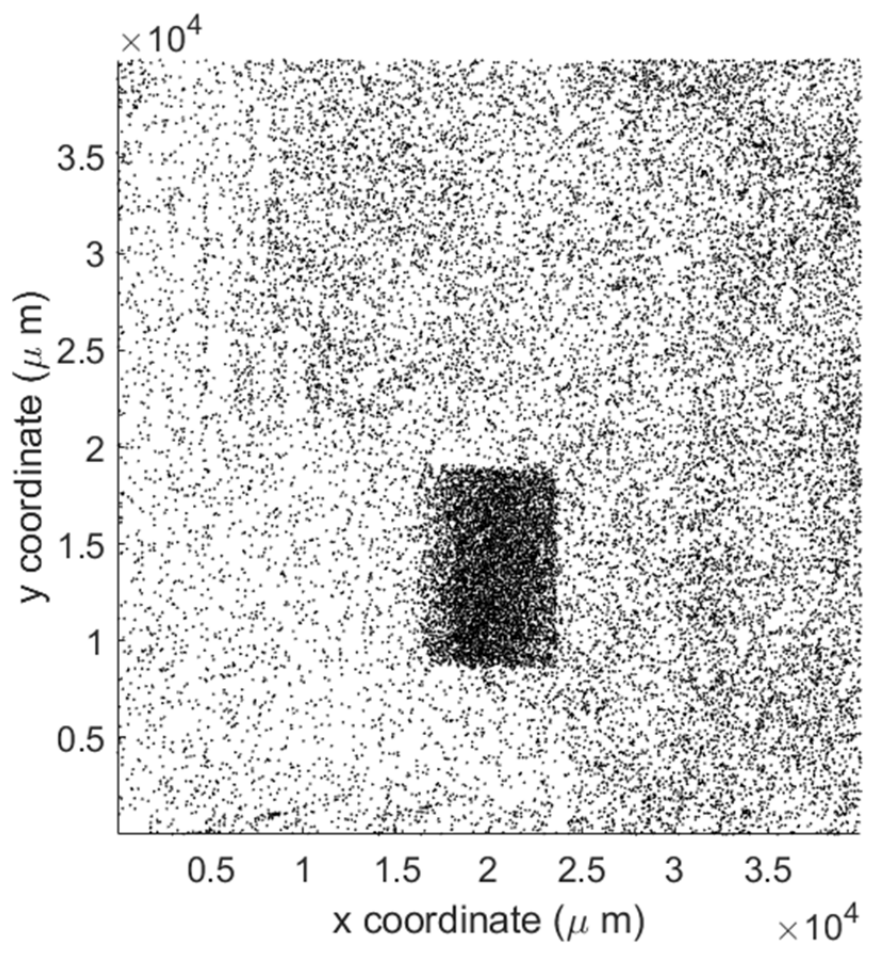

Figure 5. Muons only creating pits after moderation by the wedge.

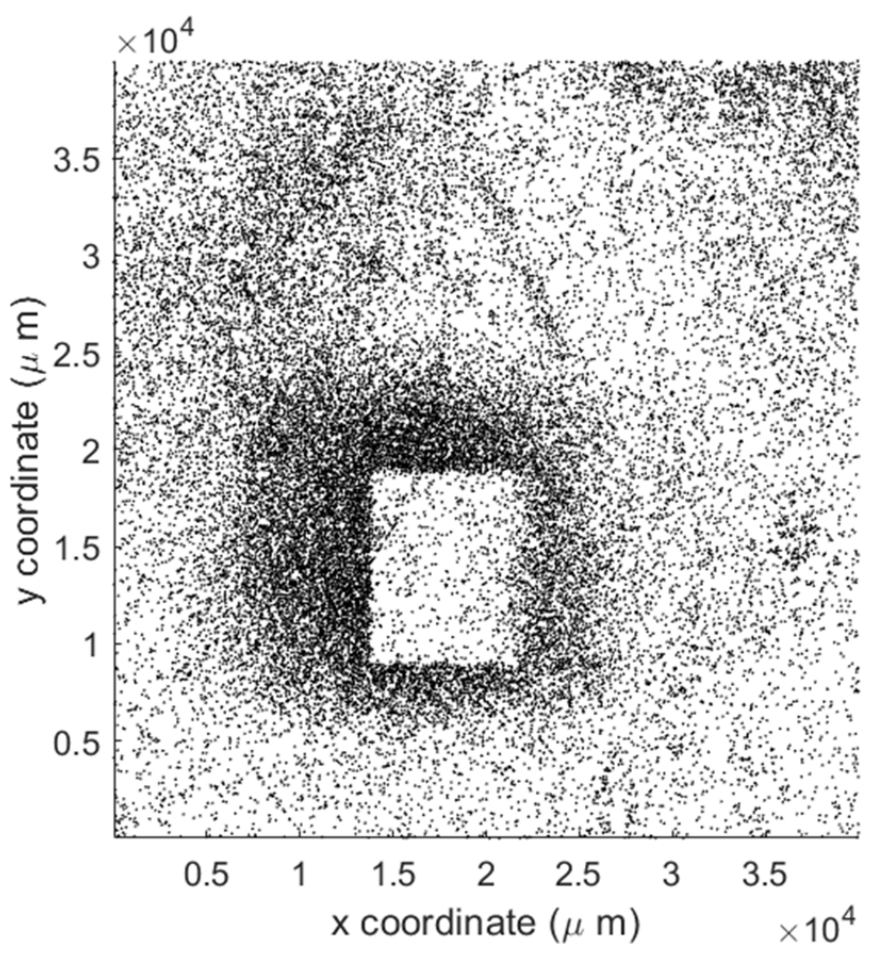

Figure 6. Muons only creating pits outside of the wedge.

$600 \mu \mathrm{m}$ of CR-39 and varying thickness of plastic wedge (max thickness $1 \mathrm{~mm}$ ).

Compare this to figure 6 , in which the majority of muons are detected outside of the imprint of the plastic wedge. This piece of CR-39 is after $800 \mu \mathrm{m}$ of CR-39, and as can be seen inside the wedge outline, most muons have already reached

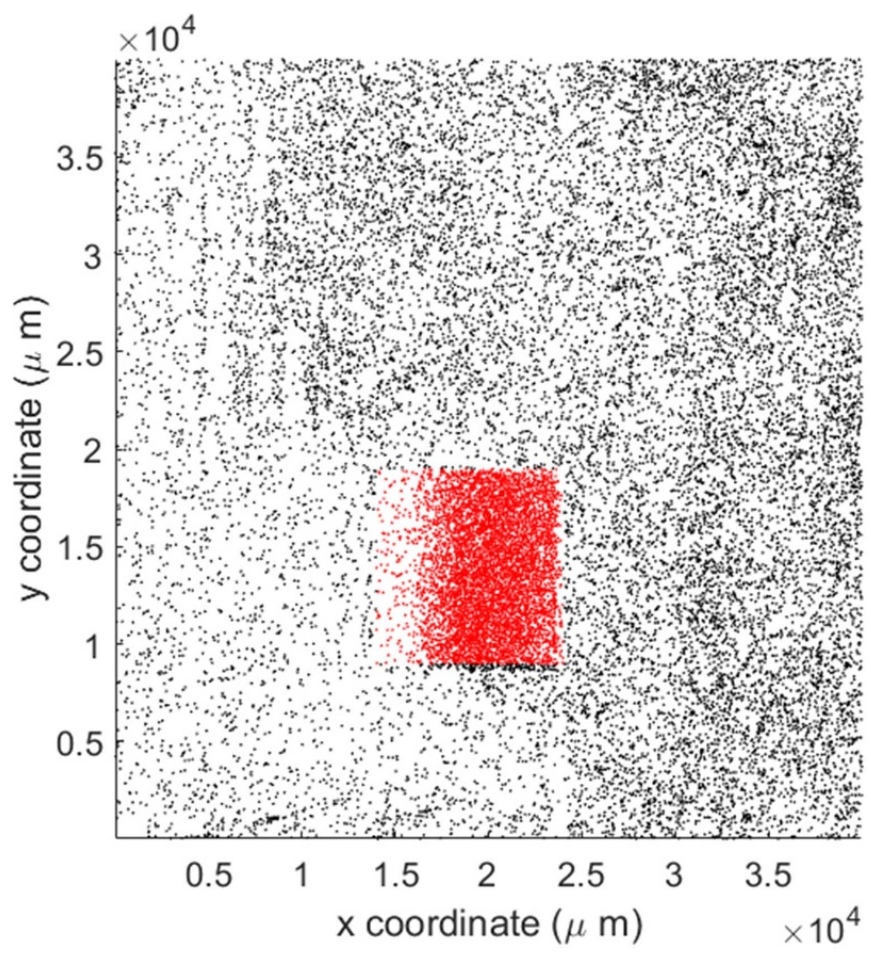

Figure 7. The wedge area of the CR-39 highlighted in red. In this stack, the wedge was plastic.

their stopping range. Muons outside of the wedge which are unmoderated are within the correct energy range to damage the CR-39 and cause pits which can be detected.

\section{Results}

Using the MuSR Spectrometer of ISIS, muons were successfully detected in CR-39 stacks. The results were analysed to determine what energy range of muons were associated with causing molecular bond damage and creating tracks within the CR-39. This was done through comparison between the scans of the pits formed on the surface of the CR-39 at different depths through the stacks, and the Geant 4 modelling results. By comparing the modelling to points across the surface of the CR-39, both inside and outside the wedge, it was deduced that muons were detected within the energy range bounded by 0 and $2.8 \mathrm{MeV}$.

To further define this energy range, a closer look within the muons moderated by a wedge could give insight. A selected piece of CR-39 was chosen (see figure 7), in which the muons pits across the wedge visually describe the optimum energy to cause detectable pits.

The area of interest beneath the wedge is shown enlarged in figure 8 , and sub-divided into 20 sections, each $50 \mu \mathrm{m}$ wide. The number of muons in each section can then be summed, as shown in figure 9, showing a maximum number of pits deposited in wedge sections $10-15$.

Using Geant4, each of the plastic wedge section median thicknesses, combined with the total CR-39 thickness $(600 \mu \mathrm{m})$ was modelled to conclude the peak energy of muons 


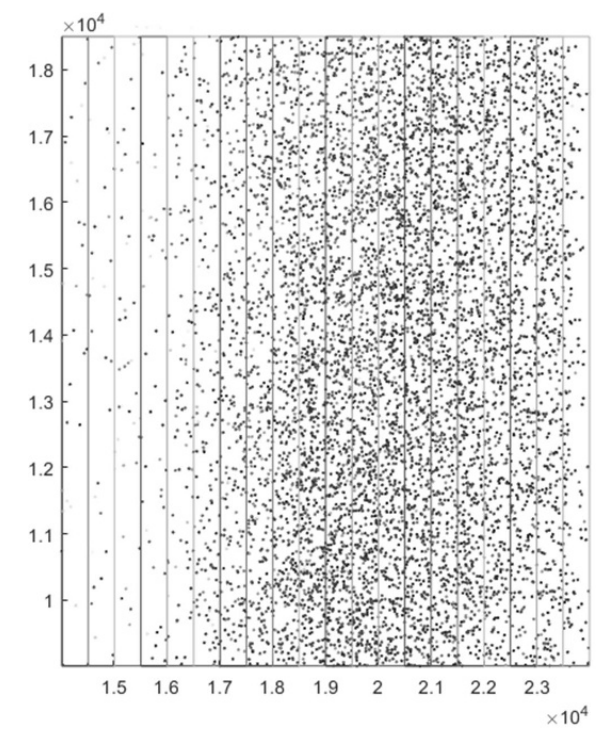

Figure 8. The wedge divided into sections through varying thickness.

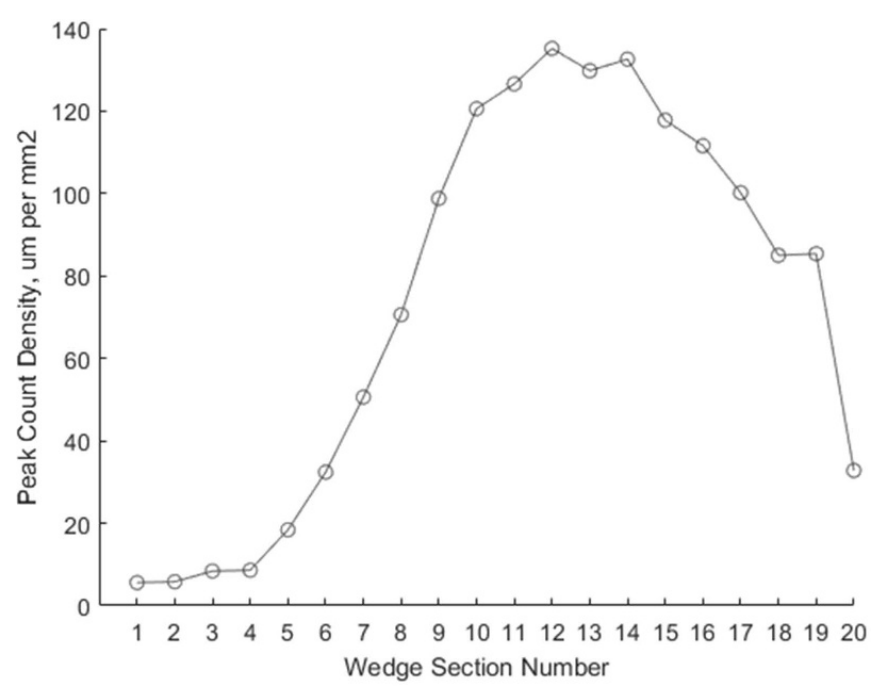

Figure 9. The pit count in each section.

reaching the CR-39 surface at that depth in the stack (see figure 10). The optimum detection energy regime for the muons is estimated in this way to be between 0.4 and $1.6 \mathrm{MeV}$.

Note that muons are detected in some wedge sections where modelling indicates none. This discrepancy is most likely due to scatter at each of the boundaries within the stack of CR-39, causing a dispersion of the muons as they traverse the stack.

The processed results were analysed to look for characteristics which could differentiate them from protons and neutrons. Figure 11 was made from one piece of CR-39, and looking at the pits created in the CR-39 by the muons across the wedge which represented differing peak energies of muons. These were plotted across the wedge to determine if there was a change in the major pit axis (the largest dimension of the pit, see figure 4) due to the change in energy of the muons. The pit

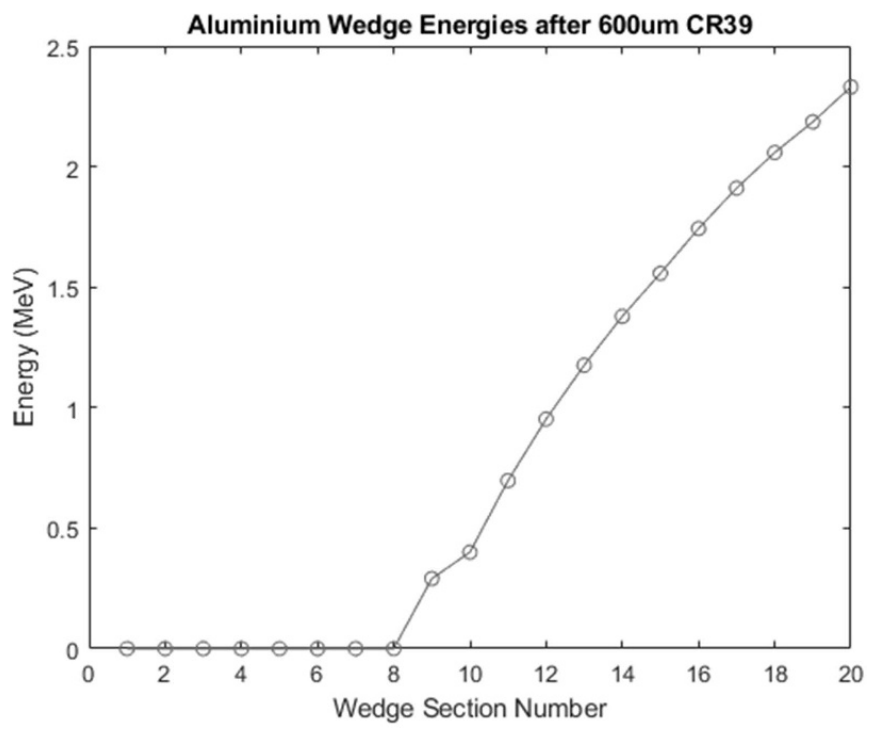

Figure 10. The peak muon energy in each section of the wedge.

size is consistent across the energies, and the dominant factor affecting the pit size is the length of time the CR-39 has been etched for, measured by the bulk etch length. At $18 \mu \mathrm{m}$ etch length, the average pit diameter is approximately $7 \mu \mathrm{m}$, and at $27 \mu \mathrm{m}$ bulk etch length, it is approximately $9 \mu \mathrm{m}$.

From a review of the literature, these pit sizes are comparable to neutron and proton pit sizes of certain energies. For $14 \mathrm{MeV}$ neutrons, with a similar bulk etch length, the pit diameter is within a similar range (2-22 $\mu \mathrm{m}$, as per [22]).

An extensive study of proton pit sizes is described in [16]. Proton pit diameters increase in size from 0.2 to $1 \mathrm{MeV}$ between $\sim 6$ and $\sim 25 \mu \mathrm{m}$, and then above $1 \mathrm{MeV}$ decrease in size with increasing energy. These pit diameters overlap with the CR-39 pit sizes described here; therefore, any possible proton sources in future experiments contributing to the pits in the CR-39 would need to be considered and controlled for.

In order to make a complete analysis, the characteristic response of CR-39 to low energy muons should also be expressed as the etch rate ratio. The etch rate ratio, $V$, is the ratio of the track etch rate $\left(V_{t}\right)$ to the bulk etch rate $\left(V_{b}\right)$ [25].

$$
V=\frac{V_{t}}{V_{b}} .
$$

In a simple approximation, perpendicular incident particles are assumed to create ideal cones for etch pits [26], and $V$ can expressed as a function of the pit radius $r$ after a bulk etch length $G$ :

$$
V=\frac{1+(r / G)^{2}}{1-(r / G)^{2}} .
$$

From our data, the etch rate ratio of CR-39 for low energy muons is estimated to be between 1.06 and 1.08. 


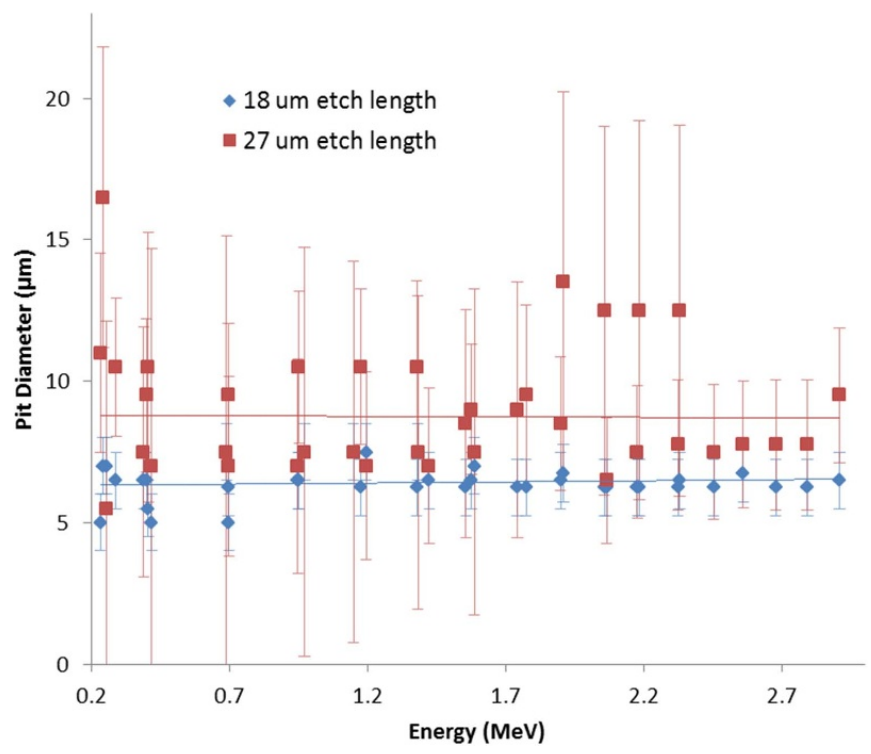

Figure 11. How the energy and etch times relate to pit diameter.

\section{Conclusions}

Monte Carlo simulations and experimental results from a muon beamline of $3.88 \mathrm{MeV}$ average energy demonstrate that SSNTDs such as CR-39 can be used to detect muons. The Monte Carlo simulations indicate that CR-39 is capable of detecting muons with energies up to $2.8 \mathrm{MeV}$, with an optimum sensitivity within the energy range of $0.4-1.6 \mathrm{MeV}$. These results support the observations of SSNTDs being effective at detecting cosmic ray muons but also offer a larger and more straightforward muon dataset due to the use of a dedicated muon source. The diameters of the pits deposited in CR-39 after exposure to muons, after being step-etched and scanned, did not strongly correlate to the energy of the muon at that pit depth, rather the diameters showed stronger correlation with the bulk etch length. CR-39's insensitivity to electrons and ionising photons enables it to be used to discriminate muons from them, in addition to its more common use as a proton or neutron detector. The potential of CR-39 to be used to discriminate tracks deposited by muons from those deposited by protons and neutrons in a similar momentum range will require a larger data set and a more comprehensive analysis. If CR-39 can be shown to be more effective in discriminating between muons and protons/neutrons in a similar momentum range it would be a highly effective muon dosimeter in environments with a high radiological dose such as laser plasma acceleration experiments.

\section{Data availability statement}

The data that support the findings of this study are available upon reasonable request from the authors.

\section{Acknowledgments}

One of the authors, David Neely, sadly passed away before this work was published.

The authors would like to express their gratitude to UKRI and STFC for providing access to the MuSR spectrometer muon beamline at the ISIS Neutron and Muon Source, UK.

\section{ORCID iD}

H S P Thomas (D) https://orcid.org/0000-0002-8176-535X

\section{References}

[1] Giacomelli P 2002 The CMS muon detector Nucl. Instrum. Methods Phys. Res. A 478 147-52

[2] Durham J M et al 2015 Tests of cosmic ray radiography for power industry applications $A I P A d v .5067111$

[3] Mangles S et al 2004 Monoenergetic beams of relativistic electrons from intense laser-plasma interactions Nature $431535-8$

[4] Geddes C G R et al 2004 High-quality electron beams from a laser wakefield accelerator using plasma-channel guiding Nature 431 538-41

[5] Faure J et al 2004 A laser-plasma accelerator producing monoenergetic electron beams Nature $431541-4$

[6] Titov A et al 2009 Dimuon production by laser-wakefield accelerated electrons Phys. Rev. ST Accel. Beams 12111303

[7] Rao B S et al 2018 Bright muon source driven by GeV electron beams from a compact laser wakefield accelerator Plasma Phys. Control. Fusion 60095002

[8] Serafini L et al 2018 A muon source based on plasma accelerators Nucl. Instrum. Methods Phys. Res. A $909309-13$

[9] Sutcliffe G D et al 2016 A novel method to recover DD fusion proton CR-39 data corrupted by fast ablator ions at OMEGA and the National Ignition Facility Rev. Sci. Instrum. $8711 \mathrm{D} 812$

[10] Sinenian N et al 2011 The response of CR-39 nuclear track detector to 1-9 MeV protons Rev. Sci. Instrum. 82103303

[11] Tanner R J et al 2005 Operational and dosimetric characteristics of etched-track neutron detectors in routine neutron radiation protection dosimetry Radiat. Meas. 40 549-59

[12] Fews A P and Henshaw D L 1982 High resolution alpha particle spectroscopy using CR-39 plastic track detector Nucl. Instrum. Methods Phys. Res. 197 517-29

[13] Jeong T W et al 2017 CR-39 track detector for multi-MeV ion spectroscopy Sci. Rep. 72152

[14] Hillier A D et al 2019 Muons at ISIS Phil. Trans. R. Soc. A 37720180064

[15] Bardin G et al 1984 A new measurement of the positive muon lifetime Phys. Lett. B 137 135-40

[16] Seimetz M et al 2018 Spectral characterization of laser-accelerated protons with CR-39 nuclear track detector Rev. Sci. Instrum. 89023302

[17] Kanasaki M et al 2016 The precise energy spectra measurements of laser-accelerated $\mathrm{MeV} / \mathrm{n}$-class high-Z ions and protons using CR-39 detectors Plasma Phys. Control. Fusion 58034013

[18] Rojas-Herrera $\mathbf{J}$ et al 2015 Impact of $\mathrm{x}$-ray dose on the response of CR-39 to $1-5.5 \mathrm{MeV}$ alphas Rev. Sci. Instrum. 86033501

[19] Sinha D et al 1997 Effect of gamma rays on PADC detectors Radiation Measurements International Conference on 
Nuclear Tracks in Solids vol 28 (Cairo, Egypt, 1-5 September 1996) pp 145-8

[20] Zaki M F and Seddik U 2007 Changes produced in etching parameters of CR-39 by high-dose pre- and post- $\gamma$ irradiation Radiat. Eff. Defects Solids 162 449-53

[21] The TASLIMAGE Radon dosimetry system (Track Analysis Systems Ltd) (Available at: www.tasl.co.ukradon.php) (Accessed 8 June 2021)

[22] Tsuruta T et al 1992 Experimental study of CR-39 etched track detector for fast neutron dosimetry J. Nucl. Sci. Technol. 29 1108-15
[23] Chiriacescu A and Lazanau I 2017 Measurement of low energy component of the flux of cosmic rays using nuclear track detectors (arXiv:1709.09116 [physics.ins-det])

[24] Manzoor S 2007 Improvements and calibrations of nuclear track detectors for rare particle searches and fragmentation studies PhD Thesis, University of Bologna \& CIIT

[25] Hassan N M et al 2013 On the mechanism of the sensitization of PADC (poly(allyl diglycol carbonate)) track detectors by carbon dioxide treatment Radiat. Meas. 59 23-9

[26] Lengar I et al 2003 Measurement of the variable track-etch rate of hydrogen, carbon and oxygen ions in CR-39 Proc. Int. Conf. Nuclear Energy for New Europe 